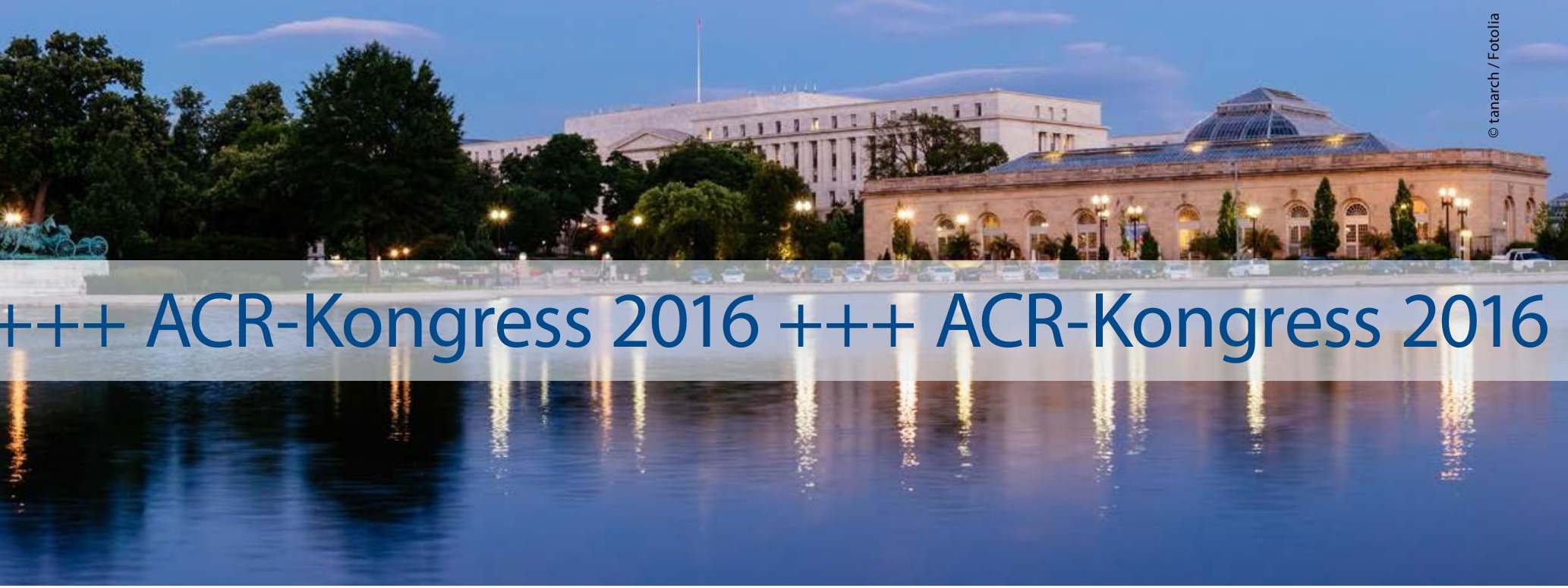

\section{Bei Rheumakranken auch aufs Herz achten}

D ass Patienten mit rheumatoider Arthritis (RA) unabhängig von der Therapie ein erhöhtes Risiko für kardiovaskuläre Ereignisse haben, ist bekannt. Weniger bekannt ist, dass die Gefahr, binnen 15 Jahren ein solches Ereignis zu erleiden, ebenso hoch ist wie das von Typ-2-Diabetikern und damit doppelt so hoch wie jenes der Allgemeinbevölke-

\section{PsA-Patienten profitieren von Statinen}

$\mathrm{G}$ ute Gründe, insbesondere Patienten mit Psoriasis Arthritis (PsA) auf Statine einzustellen, gab es schon bisher. Nun wurde dies durch eine Studie bei Patienten mit Spondylarthritiden (PsA und ankylosierende Arthritis [AS]) untermauert. In einer britischen Kohortenstudie zur Reduktion der Sterblichkeit von jeweils knapp 3.000 PsA- und ASPatienten unter respektive ohne StatinTherapie fand sich eine um 33\% reduzierte Gesamtsterblichkeit während es mittleren Follow-ups von 5,3 Jahren [Abstract 910]. Verantwortlich scheinen die dualen Effekte von Statinen im Sinne einer antientzündlichen und lipidsenkenden Wirkung zu sein, so die Autoren in der „Plenary Session I: Discovery $2016^{\prime \prime}$.

wk rung. Nach der Korrektur für traditionelle Risikofaktoren betrug die Inzidenzrate bei etablierter RA 3,6 pro 100 Personenjahre, wie in der Sitzung "Rheumatoid Arthritis - Clinical Aspects II: Risk and Impact of Comorbidity" berichtete wurde.

Die Notwendigkeit eines generellen kardiovaskulären Risikomanagements bei
RA-Patienten unterstreicht auch eine Analyse von Daten aus dem deutschen RABBIT-Register, die in der Sitzung „Rheumatoid Arthritis - Small Molecules, Biologics and Gene Therapy: Safety and Cost Effectiveness" vorgestellt wurde. Danach sind unbehandelte kardiovaskuläre Erkrankungen und vorangegangene schwere unerwünschte Ereignisse (zum Beispiel Infektionen) für RAPatienten Risikofaktoren für einen Schlaganfall.

\title{
Aktualisierte ASAS/EULAR-Leitlinie zum Management der axSpA
}

$\mathrm{D}$ as Verständnis des Krankheitsbildes axiale Spondyloarthritis (axSpA) und das Therapiespektrum haben sich geändert. Die Aktualisierung der Leitlinie von ASAS (Assessment of Spondylo-Arthritis international Society) und EULAR (European League Against Rheumatism) trägt dem Rechnung. Sie fasst nun beide Stadien - die etablierte Form der ankylosierenden Spondylitis (AS) sowie das frühe Stadium der nicht röntgenologischen axSpA (nr-axSpA) - zusammen, wie Prof. Désirée van der Heijde, Universitätsklinikum Leiden, Niederlande, berichtete. Neu ist, dass zum Monitoring nun erstmals von Patienten berichtete Ergebnisse (patient reported outcomes [PROs]) genutzt werden sollen, dass das Anstreben vorgegebener Behandlungsziele (treat to target
[T2T]) empfohlen wird und dass der Arzt Patienten zum Rauchverzicht raten soll. Zudem ist nun präziser definiert, wann eine Dauertherapie mit NSAR infrage kommt. Voraussetzung ist einerseits ein gutes Ansprechen auf die entsprechenden Medikamente, andererseits dasAuftreten von Symptome ohne NSAR. Von der Langzeitgabe systemischen Steroide wird nun bei axSpA abgeraten.

Eine weitere Neuerung betrifft die Biologika. Erfordert die Krankheitsaktivität die Behandlung mit einem biologischen DMARD, kann nun alternativ zu einem TNF-a-Inhibitor ein IL-17-Inhibitor eingesetzt werden. Bei sekundärem Wirkverlust kann nun auf einen anderen TNF-a-Hemmer oder auf einen IL17-Inhibitor gewechselt werden. $\quad w k$ 